\title{
Performance of a Structured IRA Code on a Perpendicular Recording Channel with Media Noise
}

\author{
Yang Han and William E. Ryan, \\ Department of Electrical and Computer Engineering \\ University of Arizona \\ Tucson, AZ 85721 \\ \{yhan,ryan\}@ece.arizona.edu
}

\begin{abstract}
We examine the performance of a rate-0.9 structured irregular repeat-accumulate (S-IRA) code on a perpendicular magnetic recording (PMR) channel with media noise. To account for the presence of data-dependent media noise, a pattern-dependent-noise-predictive (PDNP) channel detector is employed, in which the prediction filters are trained using the LMS algorithm. We compare the error rate performance of the S-IRA code with that of a Reed-Solomon (RS) code of the same length and rate, with the PDNP detector present in both cases. Our results show that the S-IRA code achieves a gain of over 1 dB relative to the $R S$ code for sector error rates down past $10^{-4}$, but only if the columns of its $\mathrm{H}$-matrix are properly permuted. We have also estimated that the linear density gain attainable by the S-IRA code relative to the RS code is about $8 \%$ for the operating point we have chosen.
\end{abstract}

\section{INTRODUCTION}

Low-density parity-check (LDPC) codes have been demonstrated to have near-capacity performance on many channels in the past decade [1][2][3]. Because of this, their potential has been studied for many applications, including wireless communication channels [4][5] and magnetic recording channels [6]-[11]. Their effectiveness for magnetic storage is particularly challenging due to: the binary-input constraint, severe intersymbol interference, colored noise, including patterndependent noise, and a requirement for a very low sector error rate.

In the present paper, we examine the applicability of LDPC codes to magnetic storage via computer simulations using a perpendicular recording model with pattern-dependent media noise. The application of LDPC codes to perpendicular magnetic recording has been studied in prior works [8]-[12], but none of these papers contain all of the following components:

- a length $N=4551$, dimension $K=4096$, rate-0.9 LDPC code that is a structured irregular repeat-accumulate (SIRA) code

- comparison to a Reed-Solomon (RS) code of the same length and rate, both in terms of SNR gain and user density gain

- a PR1 partial response equalization target

- a pattern-dependent noise-predictive (PDNP) soft-output partial response detector

This work was funded by a grant from Broadcom.
- a discussion of the need for an interleaver or, equivalently, permuted columns in the code's parity-check matrix $\mathbf{H}$.

- media-noise percentages of 5\%, 50\%, and 95\%

The results in this paper allow us to evaluate the potential gain offered by an LDPC code relative to a RS code. It also demonstrates the importance of the proper ordering of $\mathbf{H}$ matrix columns, an issue also considered in [13].

The paper is organized as follows. In Section II, we describe the discrete-time perpendicular recording channel model and the receiver front end. In Section III, we discuss the prediction filter design and the soft-output PDNP channel detector that attempts to whiten the noise. In Section IV, we give some details about the S-IRA code we used and discuss the importance of $\mathbf{H}$ matrix column permutations. In Section $\mathrm{V}$, we compare the performance of the S-IRA code with the RS code under different media noise conditions. Section VI presents some concluding remarks.

\section{Perpendicular Magnetic Recording Channel MODEL}

The discrete-time channel model used in this paper is an adaptation to perpendicular recording of the Lorentzian channel model by J. Moon [14]. To derive the discrete-time model, consider the continuous-time model in Fig. 1. The transition response is given in [15] by $h(t, w)=V_{\text {max }} \cdot \operatorname{erf}\left\{\frac{0.954 t}{w}\right\}$, where $V_{\max }$ is its peak amplitude $\left(-V_{\max }\right.$ is its minimum amplitude) and $w=T_{50}$ is the width of the transition response measured from $-V_{\max } / 2$ to $V_{\max } / 2{ }^{1}$ Thus, $h(t, w)$ is the response of the channel to the transitions in the recorded bits, representable as $\frac{1}{2}\left(a_{k}-a_{k-1}\right) \in\{0, \pm 1\}$, where the code bits $a_{k} \in\{-1,1\}$; hence, the presence of the discrete-time differentiator $(1-D) / 2$ in Fig. 1. The transitions suffer from random fluctuations in position $\left(\Delta t_{k}\right)$ and width $\left(\Delta w_{k}\right)$, so that the readback signal has the form

$r(t)=\sum_{k} \frac{1}{2}\left(a_{k}-a_{k-1}\right) h\left(t-k T_{c}-\Delta t_{k}, w+\Delta w_{k}\right)+e(t)$,

where $T_{c}$ is the channel bit spacing; $e(t)$ is additive white Gaussian (electronics) noise with power spectral density $N_{0}$;

\footnotetext{
${ }^{1}$ The continuous-time parameter $t$ here is normalized by the reciprocal of the head-medium velocity.
} 


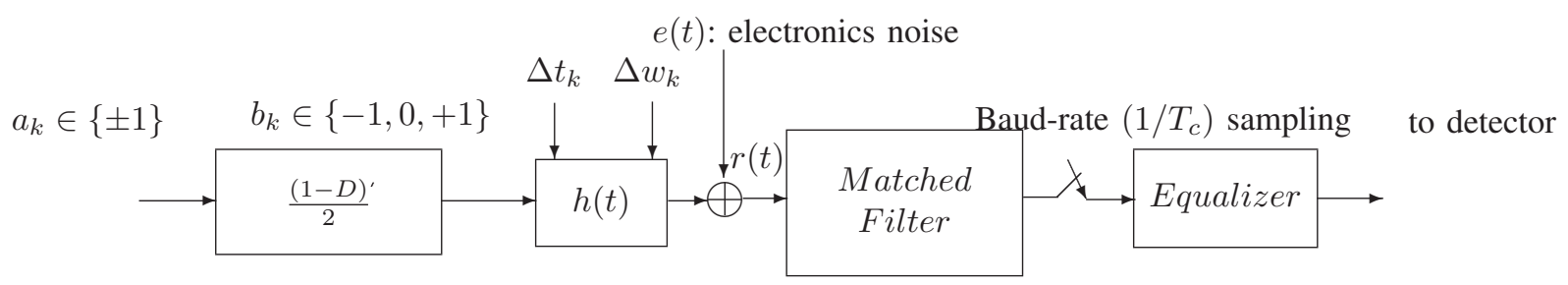

Fig. 1: Continuous-time channel model and receiver front end.

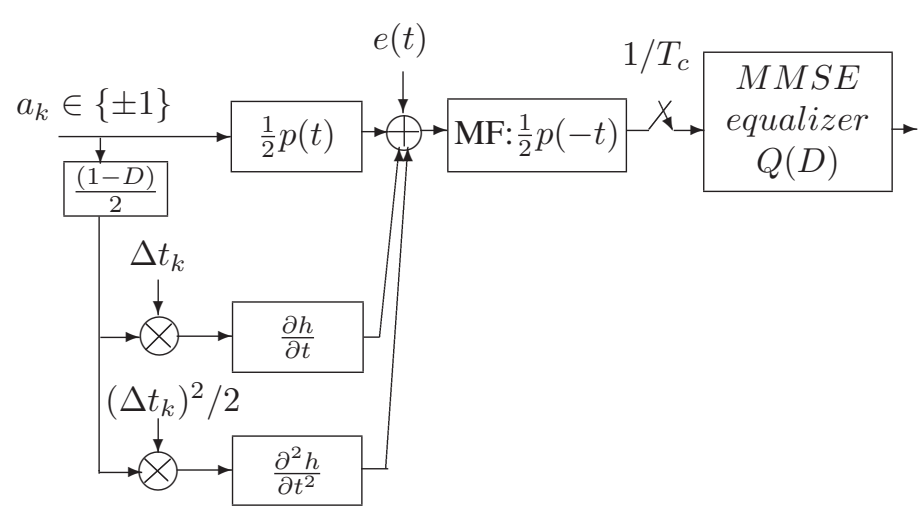

Fig. 2: Second-order continuous-time channel model.

the transition position deviation ("jitter") $\Delta t_{k}$ is modeled as truncated Gaussian $\left(\left|\Delta t_{k}\right| \leq T_{c} / 2\right)$ with variance $\sigma_{t}^{2}$; and the width deviation $\Delta w_{k}$ is single-sided Gaussian, with Gaussian variance $\sigma_{w}^{2}$. The normalized linear channel bit density is given by $D_{c}=T_{50} / T_{c}$ and the user bit density is given by $D_{u}=$ $R D_{c}=R T_{50} / T_{c}$, for code rate $R$.

By ignoring the width deviation $\Delta w_{k}$ (jitter is the dominant source of media noise) and approximating $h\left(t-k T_{c}-\Delta t_{k}, w\right)$ by three Taylor series terms, we may write

$$
\begin{aligned}
& r(t) \approx \sum_{k} a_{k} \frac{1}{2} p\left(t-k T_{c}\right) \\
& \quad+\sum_{k} \frac{1}{2}\left(a_{k}-a_{k-1}\right) \Delta t_{k} \frac{\partial}{\partial t} h\left(t-k T_{c}\right) \\
& +\sum_{k} \frac{1}{2}\left(a_{k}-a_{k-1}\right) \frac{\left(\Delta t_{k}\right)^{2}}{2} \frac{\partial^{2}}{\partial t^{2}} h\left(t-k T_{c}\right)+e(t),
\end{aligned}
$$

where $p(t)=h(t)-h\left(t-T_{c}\right)$ is the dibit response and where we dropped the dependency of $h(t, w)$ on $w$. Noting that the last three terms in (2) represent noise, the corresponding second-order channel model is shown in Fig. 2.

Let $R_{p}(D)$ denote the overall noiseless transition response from the channel input up to the sampler. The matched filter (MF) is matched to $\frac{1}{2} p(t)$ and the discrete-time partial response equalizer $Q(D)$ shapes the overall noiseless transition response into the prescribed target response $F(D)=1+D$. The equalizer is designed using the MMSE criterion, i.e., $Q(D)=F(D) /\left[R_{p}(D)+N_{0}\right]$. Let the combined discrete-time response of the first-order derivative filter and the MF be de-

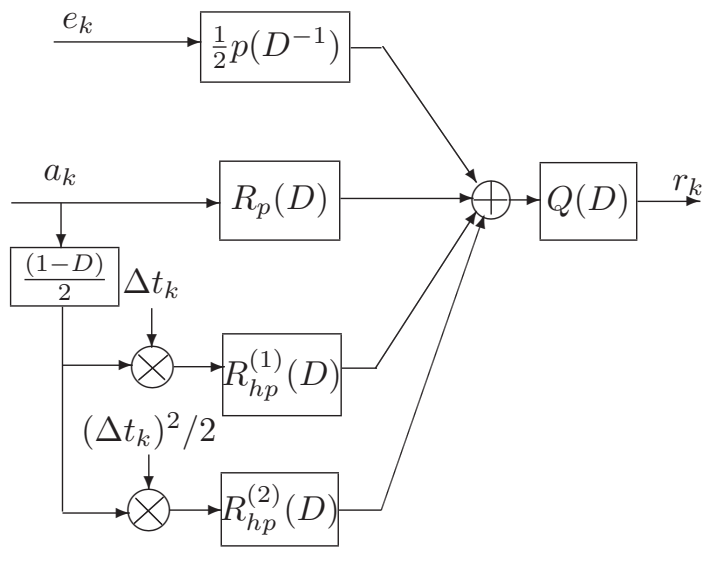

Fig. 3: Second-order discrete-time channel model.

noted by $R_{h p}^{(1)}(D)$ and let $R_{h p}^{(2)}(D)$ represent the second-order derivative filter counterpart. Then the discrete-time model for the equalizer output $r_{k}$ given channel input $a_{k}$ and electronics noise $e_{k}$ is given in Fig. 3.

The signal-to-noise ratio (SNR) in this paper is defined with respect to the electronics noise: $S N R=V_{\max }^{2} / N_{0}$. A given simulation data point will be specified as a function of this SNR and the jitter percentage, defined as

$$
J P=\frac{\sigma_{t}^{2} E_{t} / 2}{N_{0}+\sigma_{t}^{2} E_{t} / 2} \cdot 100 \%,
$$

where $E_{t}=\int\left[\frac{\partial h}{\partial t}\right]^{2} d t$. The jitter percentage is measured at the input to the equalizer. Because the second-order jitter power is much smaller than the first-order jitter power, it is not included in the JP definition.

\section{Channel Detector}

Using a standard BCJR detector designed under the AWGN assumption is far from optimal due to the presence of equalizer-colored noise and pattern-dependent noise. One straightforward way to improve the performance is to whiten the noise using noise-predictive filters which modify branch metric computations with the help of PDNP filters. A PDNP maximum-likelihood (ML) detector based on linear prediction theory for the underlying signal-dependent noise was proposed in [16]. Also in [17], the PDNP technique was applied to a belief propagation-based sub-optimal detector. In this section we review some of the principles of the PDNP-ML detector, particularly how they apply to our situation. 


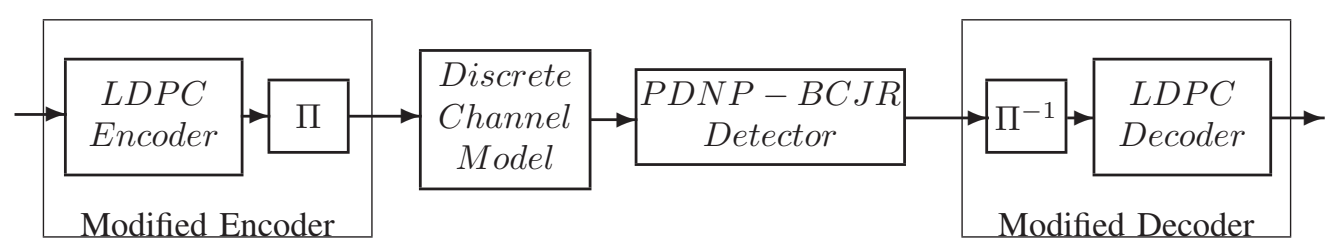

Fig. 4: System model with LDPC code.

Denote the channel input bit sequence by a, the equalized noiseless channel output sequence by $\mathbf{s}(\mathbf{a})$, and the noise sequence by $\mathbf{n}(\mathbf{a})$, all of length $N$. The equalized channel output sequence is $\mathbf{r}(\mathbf{a})=\mathbf{s}(\mathbf{a})+\mathbf{n}(\mathbf{a})$ where the $i$ th signal sample is given by $s_{i}=\sum_{j=0}^{I} f_{j} a_{i-j}$ and the equalization target $F(D)$ has $I+1$ coefficients $f_{0}, f_{1}, \cdots, f_{I}$. The ML detector finds the bit sequence a that maximizes the conditional pdf

$$
\begin{aligned}
\prod_{i=1}^{N} p\left(r_{i} \mid r_{i-1}, \cdots, r_{1} ; \mathbf{a}\right) \\
\quad=\prod_{i=1}^{N} \frac{1}{\sqrt{2 \pi} \sigma_{p}(\mathbf{a})} \exp \left\{-\frac{\left[r_{i}-s_{i}-\hat{n}_{i}(\mathbf{a})\right]^{2}}{2 \sigma_{p}^{2}(\mathbf{a})}\right\}
\end{aligned}
$$

where $\hat{n}_{i}(\mathbf{a})$ is the optimal linear prediction of the $i$ th noise sample and $\sigma_{p}^{2}(\mathbf{a})$ is the prediction error variance. From linear prediction theory, if the noise is Markov of order $Q$, it can be perfectly modeled using a $Q$ th-order autoregressive (AR) process and perfectly whitened using an optimal prediction filter with $Q$ taps. For the media noise model we are considering, though the underlying Markov order is unknown, it has been shown that the optimal predictors can be approximated using finite-length predictors of order $L$ [16]. Assuming the prediction filter taps are given by $\mathbf{q}(\mathbf{a})=\left[q_{1}(\mathbf{a}), \ldots, q_{L}(\mathbf{a})\right]$ for each bipolar $N$-sequence a, the $i$ th predicted noise sample is

$$
\hat{n}_{i}(\mathbf{a})=\sum_{l=1}^{L} q_{l}(\mathbf{a}) \cdot n_{i-l}(\mathbf{a})=\sum_{l=1}^{L} q_{l}(\mathbf{a}) \cdot\left[r_{i-l}-s_{i-l}(\mathbf{a})\right] .
$$

$\mathbf{q}(\mathbf{a})$ and $\sigma_{p}^{2}(\mathbf{a})$ can be obtained by using the Yule-Walker equation from prediction theory.

Accommodating all bipolar $N$-sequences a to realize the above algorithm in a PDNP-BCJR detector is not feasible. Thus, in practice a finite segment of $\mathbf{a}$ is utilized. Let $\tilde{\mathbf{a}}=$ $\left\{a_{i-P}, \ldots, a_{i}, \ldots, a_{i+F}\right\}$ be the finite segment of a at time index $i$, with $P$ past bits and $F$ future bits. Therefore, there are $2^{P+F+1}$ input patterns, prediction filters, and prediction error variances. $\mathbf{q}(\tilde{\mathbf{a}})$ and $\sigma_{p}^{2}(\tilde{\mathbf{a}})$ can be obtained as described above.

The resulting PDNP-BCJR detector operates on a trellis expanded from the trellis of the PR target, using (4) to calculate the branch metrics. The conventional BCJR detector has a trellis with $2^{I}$ states with each state labeled by $\left[a_{i-I}, \ldots, a_{i-1}\right]$. For the PDNP-BCJR detector, the number of states is expanded to $2^{\max (P, I+L)+F}$ and each state is labeled by $\left[a_{i-\max (P, I+L)}, \ldots, a_{i}, \ldots, a_{i+F-1}\right]$.
By solving the Yule-Walker equation for AR modeling which involves inversion of the correlation matrices obtained from real data through sample statistics, the predictor tap weights and the error variances can be obtained. But without a complete knowledge of the noise statistics and a confident noise model, matrix inversion may cause stability problems. In [16], the authors proposed a method which applies the LMS algorithm to train the predictor taps and error variances from real data.

In the LMS algorithm, the filter taps are trained by the following recursive equation:

$$
\mathbf{q}_{k+1}(\tilde{\mathbf{a}})=\mathbf{q}_{k}(\tilde{\mathbf{a}})+2 \mu \cdot e_{k}(\tilde{\mathbf{a}}) \cdot \mathbf{n}_{k}(\tilde{\mathbf{a}}),
$$

where $\mathbf{n}_{k}(\tilde{\mathbf{a}})=\left[n_{k-1}(\tilde{\mathbf{a}}), \ldots, n_{k-L}(\tilde{\mathbf{a}})\right]$ and $\mu$ is the step size that controls the convergence speed. Throughout this paper, the channel is equalized to a PR1 target $(I=1)$ and $F=1$, $P=I+L$.

For each channel condition characterized by $S N R$ and $J P$, a proper $\mu$ is chosen to make the filters converge within 400 training samples. The length of prediction filters $L$ is determined by training the filters under various channel conditions with $\mu=0.02, D_{c}=1.67$ and $L \in\{0,1, \cdots, 6\}$. We found that $L=3$ is large enough to achieve a sufficiently low MSE. Therefore, for the rest of the paper, we set $L=3$ and use $2^{I+L+F}=2^{5}$ predictors.

\section{THE S-IRA LDPC CODE}

The LDPC code-based simulation model is depicted in Fig. 4. The LDPC code we use in this paper is a rate- 0.9 $(4551,4096)$ structured irregular repeat-accumulate (S-IRA) code [18]. The nominal parity-check matrix $\mathbf{H}$ for the LDPC code has the form $\mathbf{H}=\left[\begin{array}{ll}\mathbf{H}_{1} & \mathbf{H}_{2}\end{array}\right]$, where $\mathbf{H}_{1}$ consists of an array of circulant permutation matrices and $\mathbf{H}_{2}$ is an $M \times M$ "dual-diagonal" matrix ( $M$ is the number of parity bits):

$$
\mathbf{H}_{2}=\left[\begin{array}{cccccc}
1 & & & & & \\
1 & 1 & & & & \\
& & \ldots & & & \\
& & & 1 & 1 & \\
& & & & 1 & 1
\end{array}\right] .
$$

For this code, $\mathbf{H}_{1}$ has column weight 5 .

The interleaver $\Pi$ and de-interleaver $\Pi^{-1}$ in Fig. 4 are necessary to mitigate the bursty nature of the PDNP-BCJR detector output. This characteristic can be seen in the autocovariance function of the log likelihood ratio (LLR) magnitudes at the input to the LDPC decoder. Fig. 5 shows three interleaver situations for the worst-case recorded sequence, 


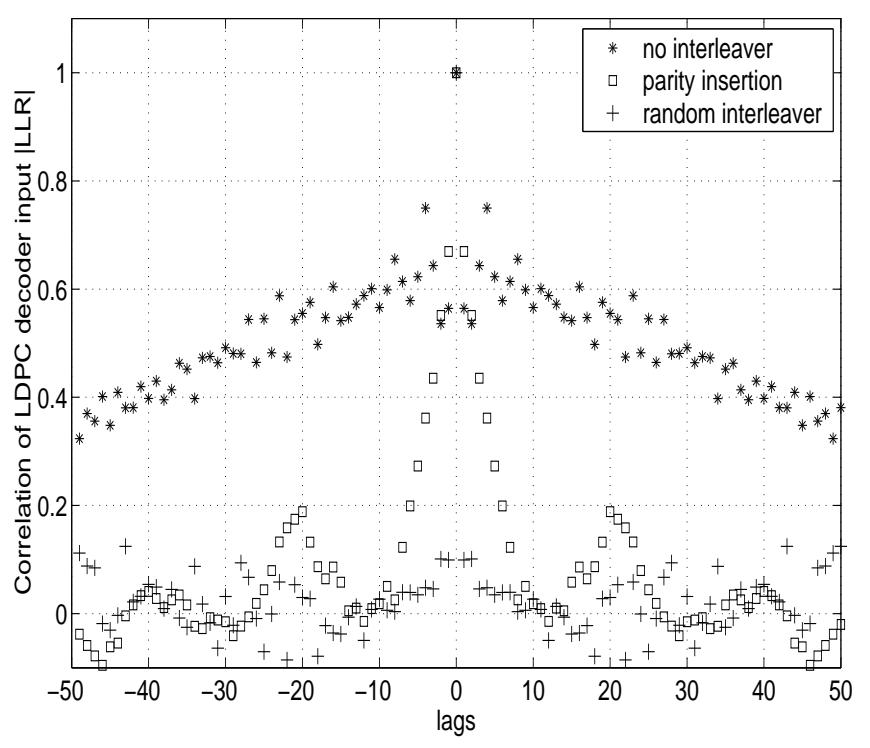

Fig. 5: Covariance functions of LDPC decoder inputs.

$\mathbf{a}=\left[\begin{array}{lllll}+1 & -1 & +1 & -1 & \ldots\end{array}\right]$, on the $95 \%$ JP channel. Our simulations used a uniform parity insertion interleaver, which spreads the parity bits evenly among the information bits with the purpose of reducing the correlations of the LDPC code's parity bits (which correspond to low weight columns). Therefore, to show the effect of this interleaver on the parity bits, Fig. 5 plots the covariance of detector output LLR magnitudes corresponding to the parity bits after de-interleaving.

The interleaver and de-interleaver are conceptual quantities and can in practice be realized by re-ordering the columns of the LDPC code parity-check matrix. It is possible to select the column permutation (equivalently, select $\Pi$ ) to accommodate both the presence of a modulation code and soft decoding [13].

A reliable way of characterizing the ability of a code to combat correlated noise or correlated fading is via the parameter $L_{\max }$ [19]. Given an LDPC code's H matrix, $L_{\max }$ is defined to be the longest erasure burst length that is guaranteed to be correctable by the iterative erasure decoding algorithm based on that matrix. For the code described above, we found that $L_{\max }=181$ erasures, an efficiency of only $181 / 455=0.39 . L_{\max }$ can be greatly improved by permuting the columns of $\mathbf{H}$ so that the "weak" parity columns (i.e., the submatrix $\mathbf{H}_{2}$ ) do not all occur together. For example, the uniform parity insertion interleaver yields $L_{\max }=239$ (efficiency 0.52). We also considered a random interleaver for which $L_{\max }=300$ (efficiency 0.66 ).

\section{Performance Results}

In this section, we present performance results on the PMR channel with recording density $D_{s}=1.67$ by simulating the second-order discrete-time model described in Section II. To make a fair comparison between the LDPC code in Section IV with RS codes, we simulate a rate- 0.9 RS code $(455,410)$ with symbols over $G F\left(2^{10}\right)$. Since the code rate is fixed to $R=0.9$ for both codes, the user density $D_{u}=1.5$.

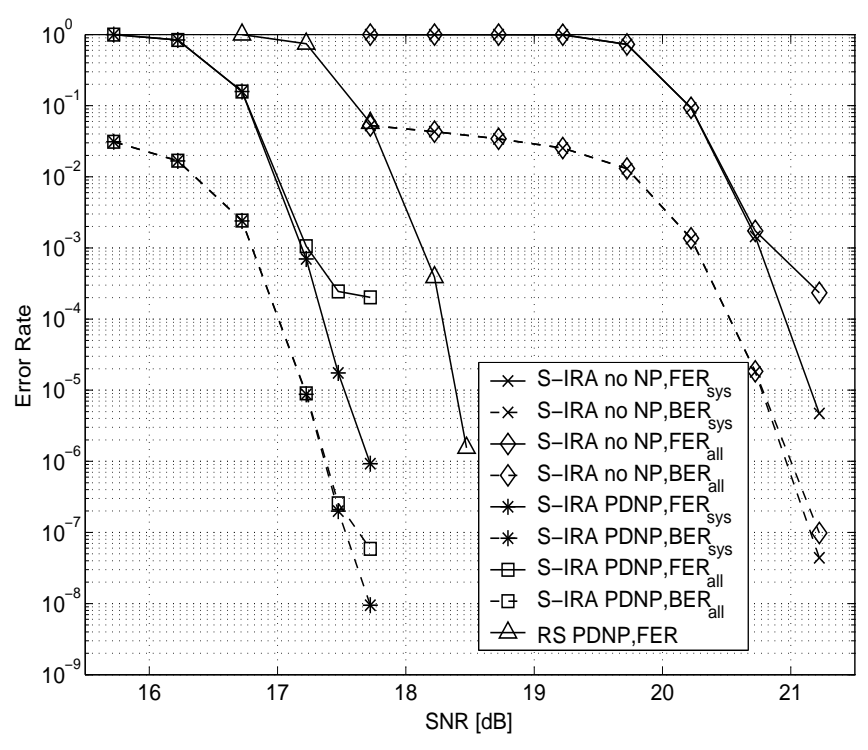

Fig. 6: Performances on PMR channel with 5\% jitter noise.

The LDPC decoder is a sum-product algorithm (SPA) decoder which updates soft information iteratively based on the modified $\mathbf{H}$ matrix corresponding to the uniform parity insertion interleaver. One can also use a more carefully designed structured column permutation for S-IRA codes [13] to achieve better performance. Although most of our results focus on the parity-insertion interleaver, later in this section we compare the performance of this interleaver to the random interleaver and to no interleaving.

As indicated in the system model of Fig. 4, we do not feed soft information back to the PDNP BCJR detector in order to maintain detector/decoder complexity to a manageable level. An additional gain on the order of $0.5 \mathrm{~dB}$ is possible if such global iterations are used, but instead we use 50 local iterations at the S-IRA SPA decoder. The performance measures used are frame error rate (FER) and bit error rate (BER), but further clarification is necessary since FER can correspond to both systematic bits and parity bits, or to only systematic bits. We denote by FER $_{\text {all }}$ the probability that a decoded code block contains one or more bit errors anywhere in the block and by FER $_{s y s}$ the probability that a decoded code block contains one or more errors among the systematic bits. The latter definition is applicable to recording devices for which an outer CRC code is available so that the LDPC information word is a CRC word. Whereas the parity bits for IRA codes are more susceptible to error than the systematic bits, this is not the case for most regular LDPC codes or for RS codes, for which $\mathrm{FER}_{\text {all }} \approx \mathrm{FER}_{\text {sys }}$. The parity bits are more susceptible to error because they correspond to the weight-two columns of $\mathbf{H}$ which have the effect also of improving the "decoding threshold" (allowing operation in lower SNR).

Figures 6, 7, and 8 present the performance results for the S-IRA and RS codes on the PMR channel with $D_{u}=1.5$ and with jitter percentages of $5 \%, 50 \%$ and $95 \%$, respectively. The parity-insertion column permutation for the S-IRA code 


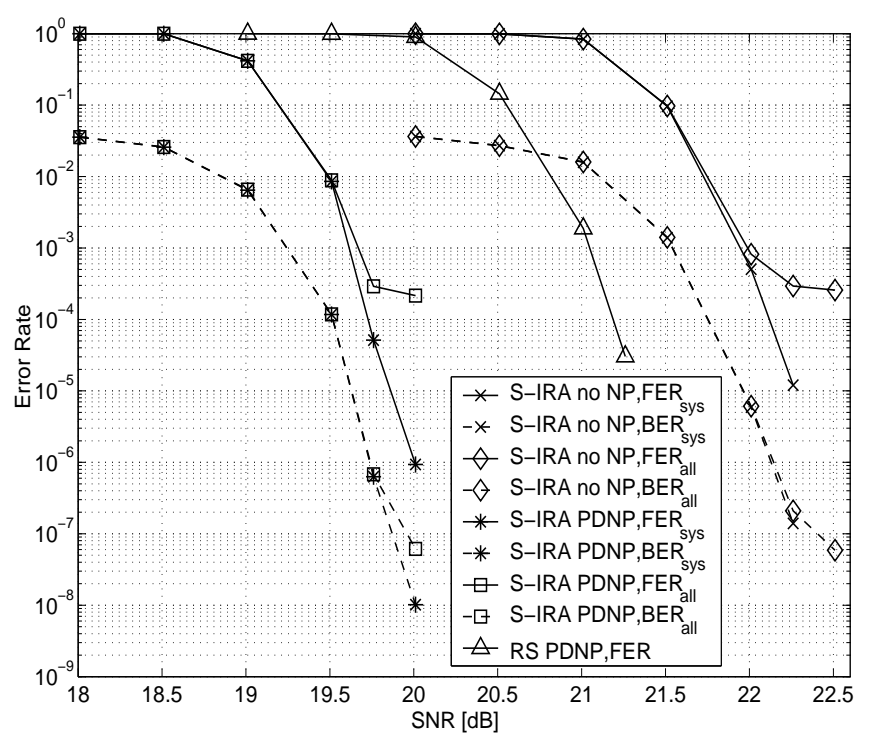

Fig. 7: Performances on PMR channel with $50 \%$ jitter noise.

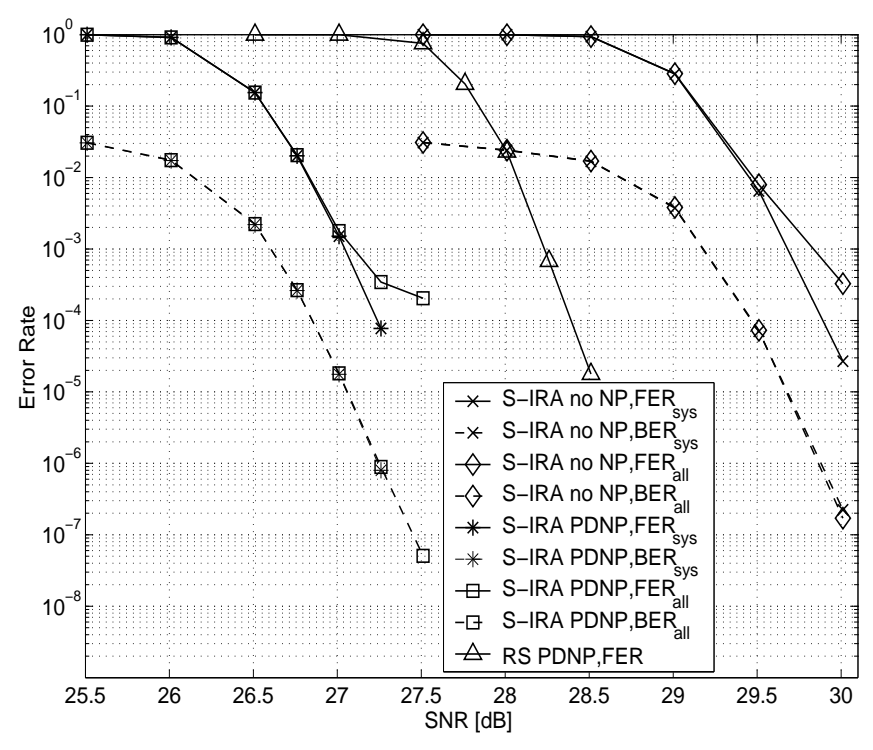

Fig. 8: Performances on PMR channel with $95 \%$ jitter noise. was used in all cases in these figures. For $5 \%$ jitter noise, we see that the gain from the PDNP-BCJR detector over the conventional non-predictive BCJR detector for the S-IRA code is about $3.7 \mathrm{~dB}$ at $\mathrm{FER}_{\text {sys }}=10^{-5}$, and the S-IRA code provides a gain of $1 \mathrm{~dB}$ over the $\mathrm{RS}$ code. With $50 \%$ jitter noise, the PDNP-BCJR detector provides a $2.4 \mathrm{~dB}$ gain at $\mathrm{FER}_{\text {sys }} \sim 10^{-5}$ over the traditional BCJR detector for the SIRA code, and the S-IRA code provides a gain of about 1.3 $\mathrm{dB}$ over the RS code at $\mathrm{FER}_{\text {sys }}=10^{-4}$. For $95 \%$ jitter noise, the gain of PDNP detector is $2.5 \mathrm{~dB}$ at FER $_{\text {sys }} \sim 10^{-4}$ and the S-IRA code offers a $1.1 \mathrm{~dB}$ gain at $\mathrm{FER}_{\text {sys }} \sim 10^{-4}$.

As observed from the figures, the PDNP-BCJR detector achieves the largest gain over the non-predictive BCJR detector with $5 \%$ jitter noise. This shows that the PDNP detector is more effective at whitening the colored noise due to electronics noise than that from jitter noise. In support of this statement, in Fig. 9 the correlation function at the detector input for the $J P=5 \%$ case has larger tails than that for the $J P=95 \%$ case. We note that the plot is based on the worse-case data sequence and the same total noise power, with electronics noise dominating in the $J P=5 \%$ case. We conjecture that increasing the detector parameters $F$ and $P$ would improve the performance of the $J P=95 \%$ case, but this will require further work.

Notice in all three figures the FER all curves suffer from a floor. Most of the floor-inducing frame errors are attributable to errors in the parity bits. The FER all error-rate floor can be lowered by using a more random $\mathbf{H}$ column permutation. In Fig. 10, we compare the performance of a computer-generated random interleaver (equivalently, column permutation) with the parity-insertion permutation and with no permutation on the $95 \%$ jitter channel. It is observed that the random permutation does indeed lower the floor significantly. Further research would be required to understand if an optimal permutation can be designed.

We remark that the S-IRA code used in this paper has a very low error-rate floor on the binary-input AWGN channel: the FER $_{\text {all }}$ curve starts to floor at $10^{-7}$ [20]. It is the presence of data-dependent correlated media noise and the bursty nature of the detector that raises the floors. The error-rate floor has been one of the major concerns regarding the application of LDPC codes in magnetic recording. However, when an outer CRC code is available so that $\mathrm{FER}_{\text {sys }}$ is the appropriate performance measure, then it appears that there is no floor down to at worst $\mathrm{FER}_{\text {sys }} \sim 10^{-6}$. (Simulating very much below this level would take many weeks.) Additionally, the floors can be improved by using a very high-rate outer RS or $\mathrm{BCH}$ code since the frame errors in the floor region usually have only a few bit errors [20]. Another approach to improve the LDPC floor is to design codes and/or permutations which yield improved performance in the presence of correlated noise.

While the results in Figures 6, 7 and 8 demonstrate that a coding gain of a least $1 \mathrm{~dB}$ is attainable by the S-IRA code relative to the RS code, it is not clear how this SNR gain translates to user density gain. Toward this end, for the $J P=95 \%$ case, we increased the user density in our S-IRA simulator until its FER $_{\text {sys }}$ curve coincided with that of the RS code. Specifically, we increased the user density $D_{u}$ from 1.5 to 1.62 and simulated the same S-IRA code again under the same channel conditions as the $95 \%$ jitter noise simulations in Fig. 8. In attempting to make a fair comparison between the two user densities, we fix the jitter noise variance $\sigma_{t}^{2}$ for the two densities for a given SNR. Since the transition response is fixed (so is $E_{t}$ ) and $J P=95 \%$, for every given SNR point, $\sigma_{t}^{2}$ can be calculated from (3). Fig. 11 shows that the S-IRA code with $D_{u}=1.62$ has comparable performance to the RS code with $D_{u}=1.5$. Hence, the $1 \mathrm{~dB}$ gain in error rate performance translates to a $(1.62-1.5) / 1.5=8 \%$ increase in user density. 


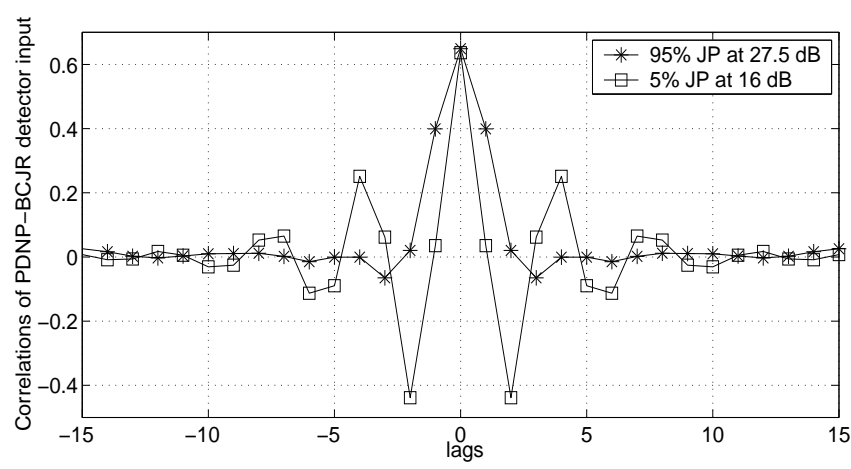

Fig. 9: Correlation functions of detector inputs.

\section{CONCLUSION}

In this paper, we evaluate the performance of a S-IRA LDPC code on perpendicular magnetic recording channel using a pattern-dependent noise-predictive BCJR detector. From simulations, we show that the S-IRA code provides an SNR gain of at least $1 \mathrm{~dB}$ which amounts to a user density gain of $8 \%$.

\section{ACKNOWLEDGMENTS}

The authors would like to thank Will Bliss and Tom Souvignier of Broadcom who directed this research, particularly Will, who found a critical error in an earlier version of the paper.

\section{REFERENCES}

[1] D. J. C. MacKay and R. M. Neal, "Near Shannon limit performance of low density parity check codes," Electron. Lett., vol. 32, pp. $1645-$ 1646, Aug. 1996.

[2] T. J. Richardson, A. Shokrollahi and R. Urbanke, "Designing of capacity-approaching irregular low-density parity-check codes," IEEE Trans. Inform. Theory, vol. 47, pp. 619-637, Feb. 2001.

[3] M. G. Luby, M. Mitzenmacher, M. A. Shokrollahi and D. A. Spielman, "Improved low-density parity-check codes using irregular graphs," IEEE Trans. Inform. Theory, vol. 47, pp. 585-598, Feb. 2001.

[4] J. Hou, P. H. Siegel and L. B. Milstein, "Performance analysis and code optimization of low density parity-check codes on Rayleigh fading channels," IEEE J. Select. Areas Commun., vol. 19, no. 5, pp. 924-934, May 2001.

[5] J. Hou, P. H. Siegel and L. B. Milstein, "Design of multi-input multioutput systems based on low-density Parity-check codes," IEEE Trans. Commun., vol. 53, no. 4, pp. 601-611, Apr. 2005.

[6] J. L. Fan, Constrained Coding and Soft Iterative Decoding for Storage, Ph.D. dissertation, Dept. Elect. Eng., Stanford Univ., Stanford, CA, Dec. 1999.

[7] W. E. Ryan, Fan Wang, R. Wood and Yan Li, "Optimal code rates for the Lorentzian channel: Shannon codes and LDPC codes," IEEE Trans. Magn., vol. 40, no. 6, pp. 3559-3565, Nov. 2004.

[8] S. Sankaranarayanan, B. Vasic and E. M. Kurtas, "Irregular low-density parity-check codes: construction and performance on perpendicular magnetic recording channels," IEEE Trans. Magn., vol. 39, pp. 25672569, Sept. 2003.

[9] E. M. Kurtas, A. V. Kuznetsov and I. Djurdjevic, "System perspectives for the application of structured LDPC codes to data storage devices," IEEE Trans. Magn., vol. 42, pp. 200-207, Feb. 2006.

[10] H. Song, R. M. Todd and J. R. Cruz, "Low density parity check codes for magnetic recording channels," IEEE Trans. Magn, vol. 36, no. 11, pp. 2183-2186, Sept. 2000.

[11] R. D. Cideciyan, E. Eleftheriou and T. Mittelholzer, "Perpendicular and longitudinal recording: A signal-processing and coding perspective," IEEE Trans. Magn, vol. 38, no. 4, pp. 1698-1704, Jul. 2002.

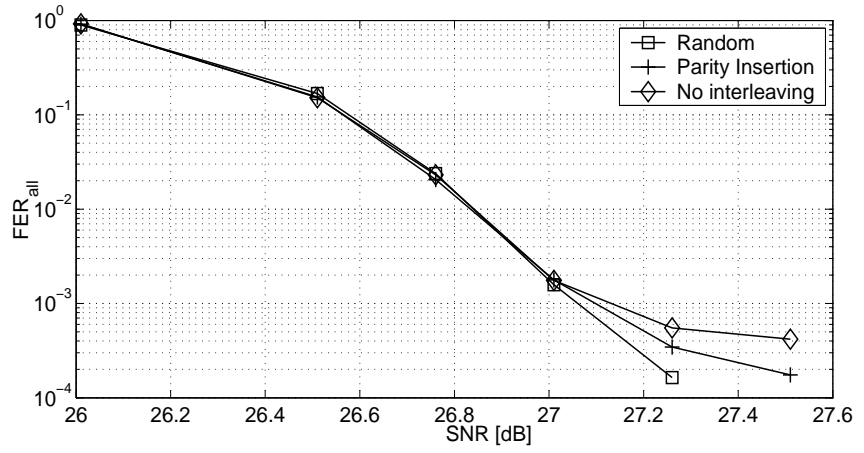

Fig. 10: The impact of various interleavings on $\mathrm{FER}_{\text {all }}$.

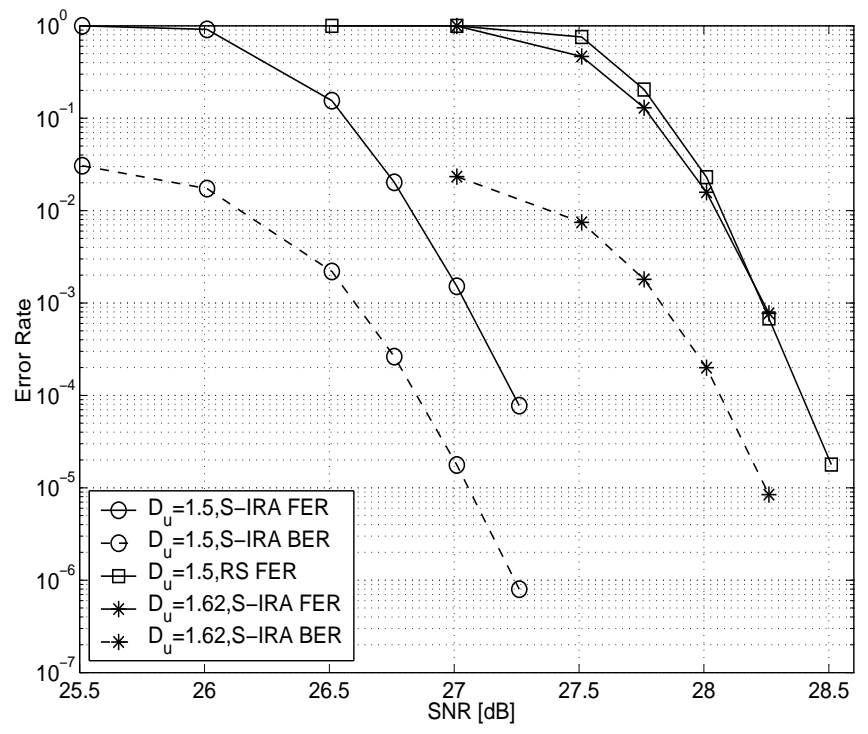

Fig. 11: The user density gain of LDPC code over RS code with $95 \%$ jitter noise.

[12] N. Nangare, Xueshi Yang, E. Kurtas and K. Narayanan, "Performance of BCJR-DFE detectors over recording channels using pattern-dependent noise prediction," IEEE Trans. Magn., vol. 41, pp. 2971-2973, Oct. 2005.

[13] Y. Han and W. E. Ryan, "Concatenating a structured LDPC code and an RLL code to preserve soft-decoding, structure, and burst correction," IEEE Trans. Magn., pp. 2558-2560, Oct. 2006.

[14] J. Moon, "Discrete-time modeling of transition-noise-dominant channels and study of detection performance," IEEE Trans. Magn., vol. 27, no. 6, Nov. 1991.

[15] P. Chaichanavong, H. Neal Bertram and P. H. Siegel, "Design Parameter Optimization for Perpendicular Magnetic Recording Systems," IEEE Trans. Magn., vol. 42, no. 10, pp. 2549-2554, Oct. 2006.

[16] J. Moon and J. Park, "Pattern-dependent noise prediction in signaldependent noise," IEEE Trans. Magn., vol. 19, no. 4, Nov. 2001.

[17] M. N. Kaynak, T. M. Duman and E. M. Kurtas, "Pattern-dependent noise predictive belief propagation," IEEE Trans. Magn., vol. 42, no. 10, Oct. 2006.

[18] Y. Zhang, W. E. Ryan, and Y. Li, "Structured eIRA codes with low floors," IEEE Proc. Int. Symp. Inf. Theory, Adelaide, Australia, 2005.

[19] F. Peng, M. Yang and W. E. Ryan, "Simplified eIRA code design and performance analysis for correlated Rayleigh fading channels," IEEE Trans. on Wireless Comm., pp. 720-725, April 2006.

[20] Y. Zhang and W. E. Ryan, "Toward low LDPC-code floors: a case study," submitted to IEEE Trans. Comm., 2007. 